ARTICLE

DOI: $10.1038 / \mathrm{s} 41467-018-04510-3$

OPEN

\title{
Budding-like division of all-aqueous emulsion droplets modulated by networks of protein nanofibrils
}

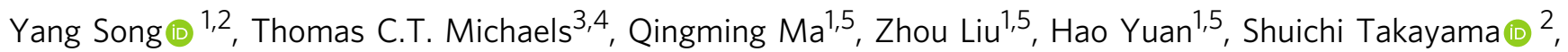
Tuomas P.J. Knowles ${ }^{3,6} \&$ Ho Cheung Shum ${ }^{1,5}$

Networks of natural protein nanofibrils, such as cytoskeletal filaments, control the shape and the division of cells, yet mimicking this functionality in a synthetic setting has proved challenging. Here, we demonstrate that artificial networks of protein nanofibrils can induce controlled deformation and division of all-aqueous emulsion droplets with budding-like morphologies. We show that this process is driven by the difference in the immersional wetting energy of the nanofibril network, and that both the size and the number of the daughter droplets formed during division can be controlled by modulating the fibril concentration and the chemical properties of the fibril network. Our results demonstrate a route for achieving biomimetic division with synthetic self-assembling fibrils and offer an engineered approach to regulate the morphology of protein gels.

\footnotetext{
${ }^{1}$ Department of Mechanical Engineering, The University of Hong Kong, Pokfulam 999077, Hong Kong. ${ }^{2}$ Wallace H. Coulter Department of Biomedical Engineering, Georgia Institute of Technology, Atlanta, GA 30332, USA. ${ }^{3}$ Department of Chemistry, University of Cambridge, Lensfield Road, Cambridge CB2 1EW, UK. ${ }^{4}$ Paulson School of Engineering and Applied Sciences, Harvard University, Cambridge, MA 02138, USA. ${ }^{5}$ HKU-Shenzhen Institute of Research and Innovation (HKU-SIRI), 518000 Shenzhen, China. ${ }^{6}$ Cavendish Laboratory, Department of Physics, University of Cambridge, JJ Thomson Avenue, Cambridge CB3 OHE, UK. Correspondence and requests for materials should be addressed to H.C.S. (email: ashum@hku.hk)
} 
T he growth of liquid protrusions from soft biological interfaces, including membranes, is a process which underlies many cellular processes, such as asexual reproduction of yeast cells ${ }^{1}$, active transport of macromolecules through endocytosis ${ }^{2}$, as well as the blebbing during the programmed cell death ${ }^{3}$. From a thermodynamic point of view, the formation of budding protrusions and their subsequent fission into daughter droplets is an energetically unfavorable processes ${ }^{4,5}$ being associated with an increase of interfacial area. The formation of surface protrusions in liquid droplet systems ${ }^{6-9}$, therefore, requires interfacial engineering by means such as incorporating proteins into the membrane surrounding the droplet $^{6,7}$, inducing the dewetting of sub-droplets from vesicles with multi-phase compartments ${ }^{8,9}$, or activating chemical reactions that destabilize the droplet interface ${ }^{10,11}$. The formation of such membrane protrusions in response to environmental stimuli $^{12}$ can lead to the complete fission of the daughter droplets.

Living cells are able to control their shape and division using networks of protein nanofibrils, such as the cytoskeleton ${ }^{13-16}$. Network-mediated cellular division, such as the condensation of the septal Z-ring ${ }^{17-19}$ in dividing bacteria, and the polymerization (or depolymerization) of actin filaments in eukaryotic cells ${ }^{20,21}$ are all related to the functioning of the protein networks at variable fibril concentrations. However, mimicking natural fibrilnetwork-mediated division remains challenging, even though this functionality could have significant applications in a synthetic setting. Synthetic cytoplasmic matrices could provide a bottomup approach ${ }^{22}$ to unravel the role of protein networks in the division of protocells. Water-in-water (w/w) emulsion droplets, formed for instance by dispensing a dextran-rich aqueous phase into an immiscible polyethylene glycol (PEG)-rich continuous aqueous phase, have been used previously to simulate compartmentalized cytoplasm ${ }^{23}, 24$. All-aqueous emulsions are particularly advantageous in this context, due to the characteristic ultralow interfacial tension ${ }^{25}\left(<1 \times 10^{-3} \mathrm{~N} \mathrm{~m}^{-1}\right)$ which dramatically lowers the energetic cost for interfacial area increase during droplet division.

In this paper, we demonstrate that the addition of protein nanofibrils to all-aqueous emulsions can induce the division of the $\mathrm{w} / \mathrm{w}$ emulsion droplets and that the concentration of fibrils controls the division regimes of budding droplets. Our observations not only provide a simplified physical model for reproducing droplet division in a synthetic setting, but also inspire engineered approaches to adjust the surface morphology of protein gels.

\section{Results}

Gelation of protein nanofibril suspensions. Protein nanofibrils were synthesized by polymerizing lysozyme monomers at $65^{\circ} \mathrm{C}$ under acidic conditions ( $\mathrm{pH}=1.6$, see Methods) ${ }^{26}$. After cooling to room temperature, the nanofibril suspension ( $2 \mathrm{wt} \%$ ) formed a soft gel. By introducing shear forces through stirring, the nanofibril gel transformed into a viscoelastic fluid (see Supplementary Fig. 1), but returned to the gel phase under quiescent conditions. The gelation of the fibril suspension could be controlled by dissolving additional solutes in the aqueous medium. For example, when the fibril suspension was injected slowly into a $10 \mathrm{wt} \%$ dextran solution, it formed a gel (see Supplementary Fig. 1d). However, when injected into a $8 \mathrm{wt} \%$ PEG solution, the fibrils remained suspended in solution without undergoing gelation, probably due to the incorporation of PEG molecules into the fibril network.

Division of $\mathrm{w} / \mathrm{w}$ drops loaded with protein nanofibrils. An aqueous suspension of $1.2 \mathrm{wt} \%$ fibrils in $7.5 \mathrm{wt} \%$ dextran T500 was dispersed into an acidic PEG ( $8 \mathrm{wt} \%, \mathrm{Mw}=20,000, \mathrm{pH}=3$ ) solution via electrospray ${ }^{27}$, resulting in the formation of dextranin-PEG w/w emulsion droplets. Due to the higher osmolality of the PEG-rich continuous phase, the droplets underwent dehydration until a balance was established between the osmolality of the dextran-rich phase and that of the PEG-rich phase. During droplet shrinking, small buds were observed to form on the droplet surface (Fig. 1a). The diameter of these buds increased over time due to coalescence; eventually, each mother droplet split into a well-defined number of daughter droplets. Similar protrusions were observed to form also on flat w/w interfaces (Fig. 1b). The formation of buds was strongly dependent on the presence of nanofibrils in the dextran-rich droplet phase: no protrusions were observed under the same conditions of osmotic pressure and $\mathrm{w} / \mathrm{w}$ interfacial tension without loading a sufficient amount of fibrils into the droplet phase (Fig. 1c, d).

Mechanism of budding-like division of $w / w$ droplets. To probe the mechanism behind the observed budding-like division of $\mathrm{w} / \mathrm{w}$ emulsion droplets, we labeled protein nanofibrils with the fluorescent dye Thioflavin $\mathrm{T}(\mathrm{ThT})^{28}$. After homogenization of the ThT-labeled fibril suspension in the dextran phase, the droplet phase was immediately electrosprayed onto the continuous phase to form $\mathrm{w} / \mathrm{w}$ emulsion droplets. Water was partially extracted from the droplet phase due to a prevailing osmotic pressure (30 mOsm kg${ }^{-1}$ ) between the two immiscible aqueous phases. Since fibrils do not permeate the $\mathrm{w} / \mathrm{w}$ interface, droplet dehydration resulted into an increase of fibril concentration in the droplet phase. Above a critical fibril concentration, a viscoelastic fibril network composed of fibril bundles could be seen to contract in response to an abrupt change in osmotic pressure and then phase-separate from the dextran-rich droplet phase (see Fig. 2a, b and Supplementary Fig. 2). The phase separation originates from the intrinsic incompatibility of the fibril network with the dextran-rich phase and the consequent need to reduce the interfacial area between the two phases. The contraction of viscoelastic fibril network pulls the droplet interface, leading to buckled interfaces (Fig. 2c). After phase separation, the fibril network underwent spontaneous dewetting from complete to partial wetting of the network in the droplet phase (Fig. 2d, e); the interface between the network and the dextran-rich phase is indeed decreased during budding, as seen in Fig. $2 \mathrm{f}$ and g. The dewetting transition is driven by (see Supplementary Note 1):

$$
\sigma_{\mathrm{PEG} / \mathrm{net}}-\left(\sigma_{\mathrm{dex} / \mathrm{net}}+\sigma_{\mathrm{w} / \mathrm{w}} \cos \theta\right)<0,
$$

where $\sigma_{\mathrm{w} / \mathrm{w}}$ is the w/w interfacial tension, and $\sigma_{\mathrm{dex} / \text { net }}$ and $\sigma_{\mathrm{PEG} / \text { net }}$ are, respectively, the interfacial tensions between the fibril network and the dextran-rich and PEG-rich phases; $\theta$ is the wetting angle formed by the dextran-rich phase and the fibril network. The driving force for dewetting is favorable when the expression in Eq. (1) is negative. This condition is satisfied in our experiments because $\sigma_{\mathrm{PEG} / \text { net }} \approx 0$ (the fibril network does not phase separate in the PEG-rich phase); moreover, under all the tested experimental conditions, we measured $\sigma_{\mathrm{dex} / \mathrm{net}}<\sigma_{\mathrm{w} / \mathrm{w}}$ (see Supplementary Fig. 3), leading to a final wetting angle of $\theta=$ $\arccos \left(-\sigma_{\mathrm{dex} / \mathrm{net}} / \sigma_{\mathrm{w} / \mathrm{w}}\right)>90^{\circ}$, in agreement with the observations of Fig. $2 \mathrm{f}$ and g. Such a large wetting angle is consistent with the higher partitioning affinity of fibrils to the PEG-rich phase compared to the dextran-rich phase (Supplementary Fig. 4$)^{29}$.

As the viscoelastic fibril network contracts under the osmotic pressure (see Supplementary Fig. 5 and Supplementary Note 2), the dextran-rich phase is squirted out of the porous fibril network, resulting in the formation of liquid protrusions with large interfacial area, in analogy to what happens when a waterabsorbing sponge is squeezed in oil. In the absence of any 

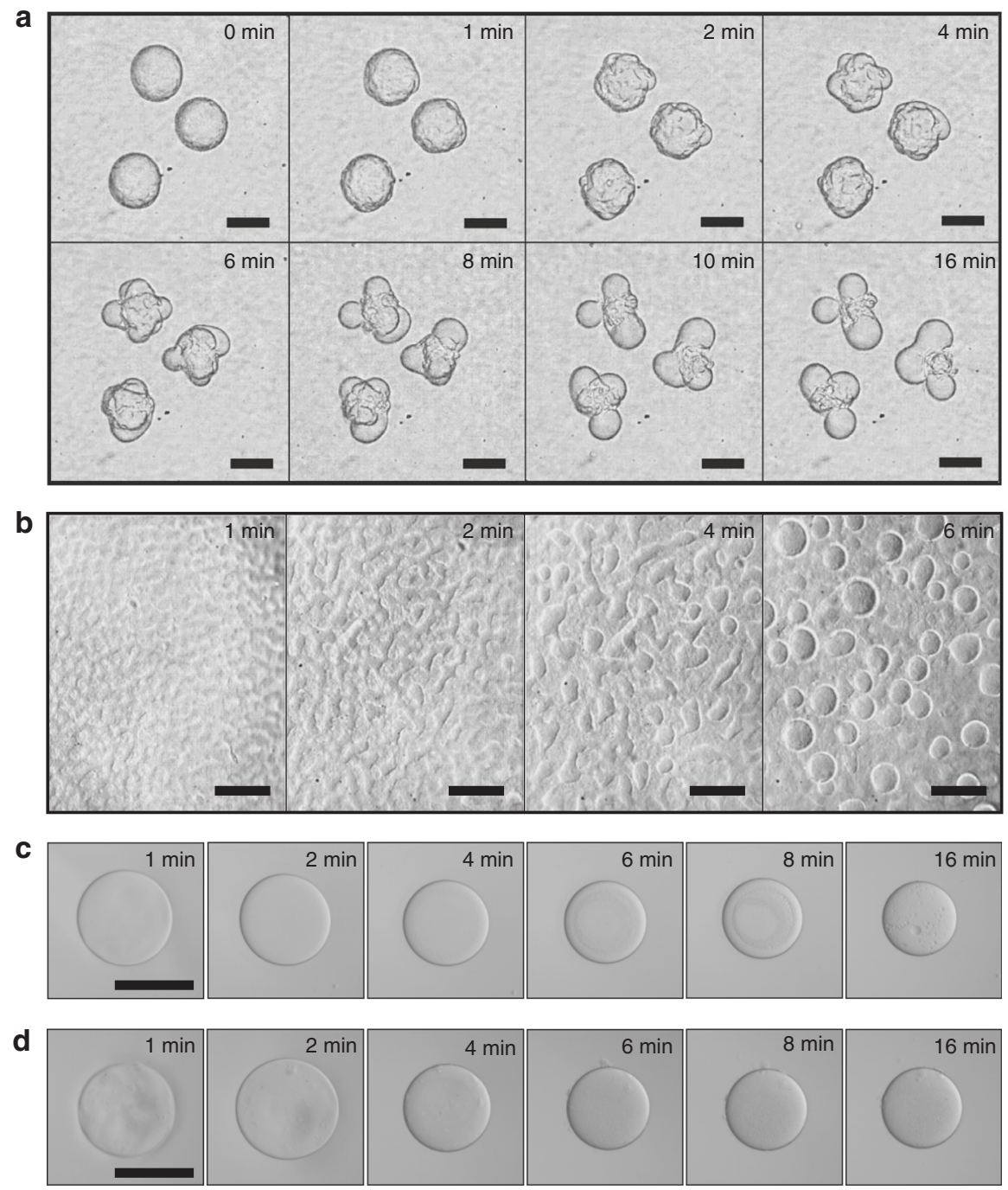

Fig. 1 Budding-like division of $w / w$ droplets loaded with protein nanofibrils. a Optical microscope images of dividing w/w emulsion droplets preloaded with protein nanofibrils. The droplet phase was preloaded with 7.5 wt\% dextran T500 and 1.2 wt\% lysozyme nanofibrils before injection into a 8 wt $\%$ PEG-10 $\mathrm{mM} \mathrm{HCl}$ solution. $\mathbf{b}$ Optical microscope images showing the formation of protrusions on a flat w/w interface. No splitting of w/w droplets was observed when the concentration of fibrils was c 0 wt $\%$ and $\mathbf{d} 0.1 \mathrm{wt} \%$. Scale bars are $200 \mu \mathrm{m}$

stabilizing mechanism, the protruded subdroplets are unstable against coalescence and their average radius, $R$, increases over time. The merging of the daughter droplets, however, can be halted by the adsorption of fibrils at the w/w interface. We have previously reported that protein nanofibrils efficiently stabilize dextran-in-PEG emulsion droplets due to the entrapment of fibrils at the $\mathrm{w} / \mathrm{w}$ interface ${ }^{29}$. If the total amount of adsorbed fibrils is insufficient to fully cover the $\mathrm{w} / \mathrm{w}$ interface, the surface protrusions coalesce in order to minimize the total interfacial area, $A \propto \frac{1}{R}$. However, as the daughter droplets coalesce, the w/w surface coverage by fibrils progressively increases in proportion to $R$ until droplet coalescence is eventually halted. In analogy to the physics of Pickering emulsions ${ }^{30}$, the average diameter $D=2 R$ of daughter droplets stabilized by adsorbed protein fibrils can be estimated as a function of the initial concentration of fibrils in the mother droplet, $C_{\text {fibril, }}$, as (see Supplementary Note 3 and Supplementary Fig. 6):

$$
D_{\text {daughter }}=2 R_{\text {daughter }}=\frac{3 a \sigma_{\mathrm{w} / \mathrm{w}}}{\left(\sigma_{\mathrm{dex} / \mathrm{net}}-\sigma_{\mathrm{PEG} / \mathrm{net}}\right) \eta C_{\mathrm{fibril}}},
$$

where $a$ is the radius of the cross-section of fibril bundles, and $\eta$ is the shrinkage ratio, i.e., the ratio between the initial volume of the mother droplet and the total volume of daughter droplets. Equation (2) predicts that the diameter of daughter droplets, $D_{\text {daughter, }}$ increases in proportion to $\sigma_{\mathrm{w} / \mathrm{w}}$ (see Fig. 3a, Supplementary Fig. 7, Supplementary Note 4, and Supplementary Table 1) and decreases in proportion to the fibril concentration, $C_{\text {fibril }}$, and the shrinkage ratio $\eta$. Interestingly, $D_{\text {daughter }}$ is not affected by variations in the volume of the mother droplets (see Fig. 3a and Supplementary Fig. 8).

Effect of fibril concentration on droplet division. According to Eq. (2), the initial concentration of fibrils in the droplet phase, $C_{\text {fibril }}$, controls the final size of the daughter droplets by determining the availability of excess fibrils that can stabilize the $\mathrm{w} / \mathrm{w}$ interface. To test this prediction, we varied systematically the concentration of fibrils in the droplet phase while leaving the other parameters unchanged. When the fibril concentration was below $0.2 \mathrm{wt} \%$, no phase separation into a fibril network was observed (Fig. 1c, d) and, as a result, droplets remained stable over time without undergoing division. When the fibril concentration was above $0.3 \mathrm{wt} \%$, a fibril network was observed to phase-separate from the dextran-rich phase after droplet 
a
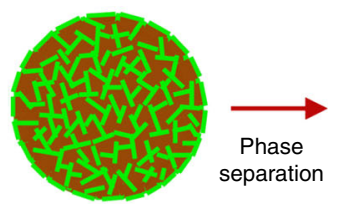

b
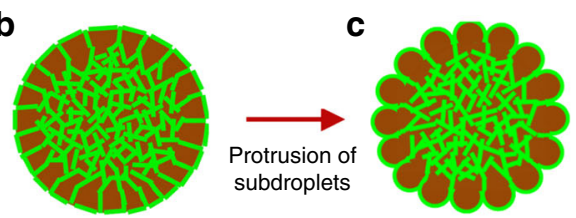

c

subdroplets
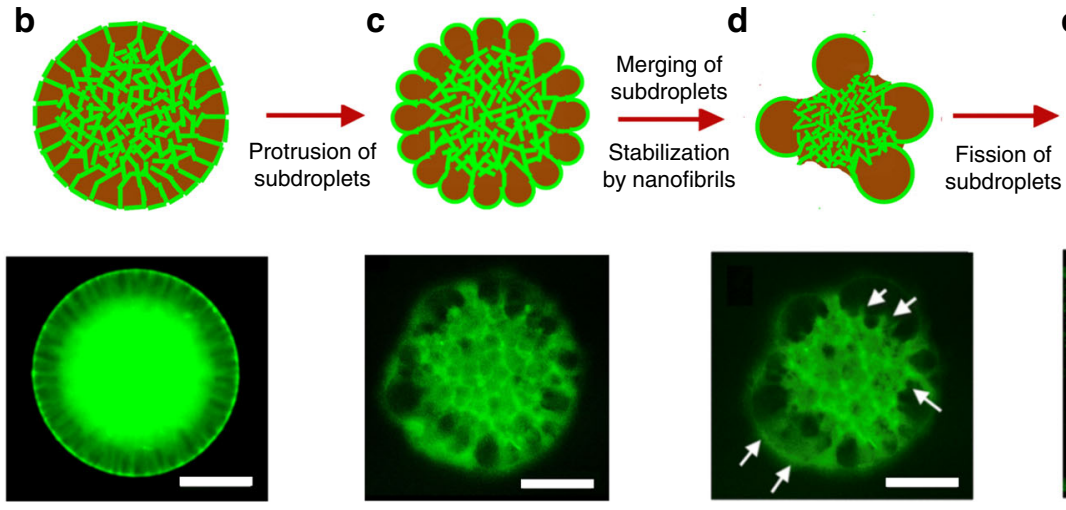

e
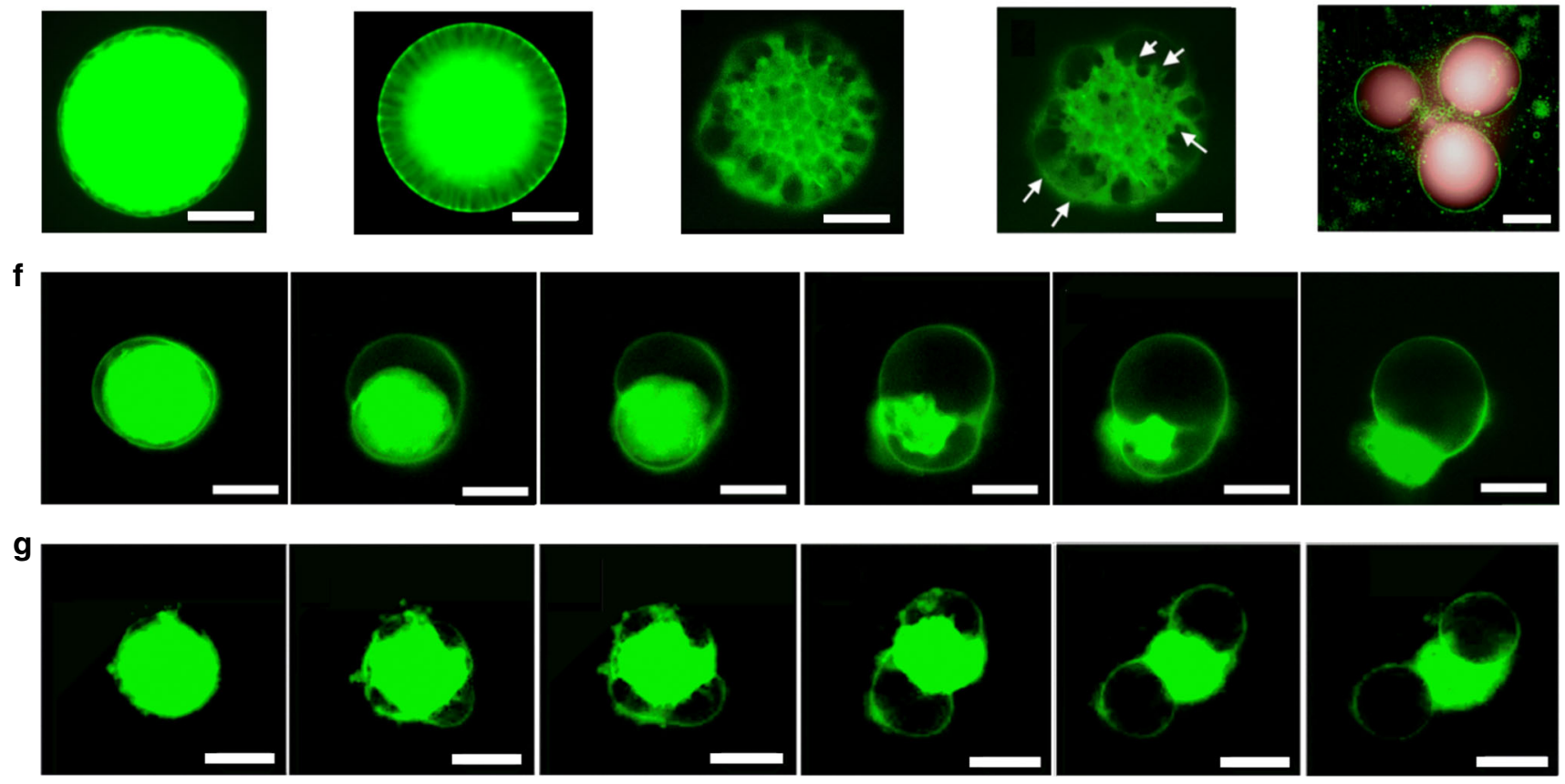

Fig. 2 Mechanism of budding-like division of w/w emulsion droplets mediated by protein nanofibrils. a-e Schematic diagram and fluorescence microscope images describing the mechanistic steps in the budding-like division of w/w droplets. The fibril network (stained green) contracts and phase-separates from the remaining liquid phase through a dewetting transition. In this transition, the as-formed protrusions coalesce (as pinpointed by the white arrows) until a sufficient amount of fibrils adsorbs at the w/w interface to stabilize daughter droplets. Complete fission of dextran-rich subdroplets (faked red color) is observed after total decomposition of the fibril networks in the PEG-rich continuous phase. Scale bars, $100 \mu \mathrm{m}$. Fluorescence microscope images showing $\mathbf{f}$ single division $\left(C_{\text {fibril }}=0.5 \mathrm{wt} \%\right)$ and $\mathbf{g}$ multiple division $\left(C_{\text {fibril }}=1.0 \mathrm{wt} \%\right)$ of $\mathrm{w} / \mathrm{w}$ droplets preloaded with protein nanofibrils and $8 \%$ dextran. Scale bars, $200 \mu \mathrm{m}$. The continuous phase consists of $8 \mathrm{wt} \%$ PEG dissolved in $10 \mathrm{mM} \mathrm{HCl}$

dehydration. Under these conditions, however, the amount of fibrils was insufficient to stabilize the surface protrusions; as a result, the daughter droplets were observed to merge into a single droplet before total decomposition of the fibril network into the PEG-rich phase (single-division regime, see Fig. 2f). When the concentration of fibrils was above a critical value $C_{\text {fibril }}^{*}(1.0 \mathrm{wt} \%)$, the formation of multiple stable protrusions was observed (multiple-division regime, Fig. 2g). In this regime, the total interfacial area of the daughter droplets was significantly larger than that of the mother droplet ${ }^{31}$. The relative increase in w/w interfacial area was found to scale linearly with initial fibril concentration (see Fig. $3 b$ and Supplementary Note 5). Furthermore, the radius of the stabilized surface protrusions was found to decrease with increasing fibril concentration (Fig. 3c), in agreement with our theoretical predictions. Finally, we measured the size distribution of dividing droplets (see Supplementary Figs. 9, 10) and found that, due to the enhanced stabilization of daughter droplets, higher fibril concentrations were associated with a higher degree of size monodispersity.

Effect of shrinkage ratio on droplet division. As a next step, we investigated the role of shrinkage ratio $\eta$ on droplet division. To do so, we reduced the concentration of dextran in the droplet phase from 13 to $4 \mathrm{wt} \%$, while maintaining the initial fibril concentration and the PEG concentration in the continuous phase constant. With a lower concentration of dextran in the droplet phase, a higher osmotic pressure is created to extract water from the droplet and induce budding (Supplementary Fig. 11). This resulted in a higher degree of dehydration and, consequently, a denser fibril network. The average diameter of the daughter droplets was found to decrease with increasing shrinkage ratio $\eta$ (Fig. 3d), in agreement with the prediction of Eq. (2). The experiment was performed for two different fibril concentrations. Combining the effect of shrinkage ratio, $\eta$, and initial fibril concentration, $C_{\text {fibril }}$, we define a combined parameter $X=$ $\eta C_{\text {fibril }}$ that reflects the fibril concentration after dehydration of the mother droplet. The average radius of the daughter droplets was found to decrease inversely proportional to this combined parameter $X$, confirming that the final diameter of the stabilized dextran-rich buds can be equally modulated by changing either the initial fibril concentration or the droplet shrinkage ratio (Fig. 3d, e and Supplementary Note 6).

Fabrication of protein microgels with surface protrusions. The budding-like division of w/w emulsion droplets using protein nanofibrils demonstrated in this paper can be applied to induce surface protrusions in protein hydrogels. We have previously reported on a microfluidic approach to generate all-aqueous emulsions and jets ${ }^{32}$. By incorporating fibril networks into the dispensing phase, we could induce small protrusions on the surface of the droplets and jets. The dextran-rich buds could be solidified by means of osmotic dehydration in the continuous PEG phase ( $>20 \%)$, and the speed of dehydration increased with the PEG concentration (see Methods ${ }^{33}$ ). By balancing the speed 

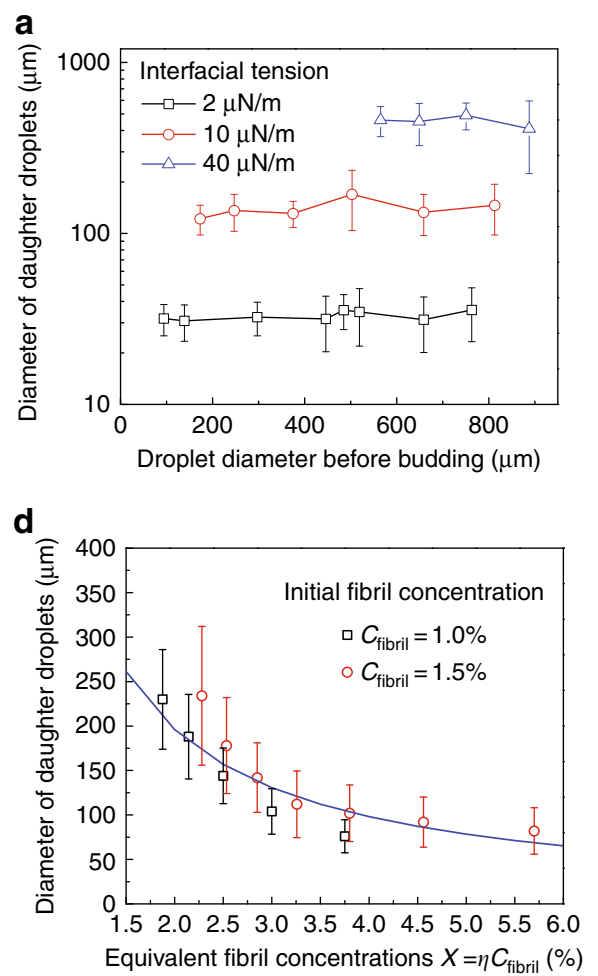

b

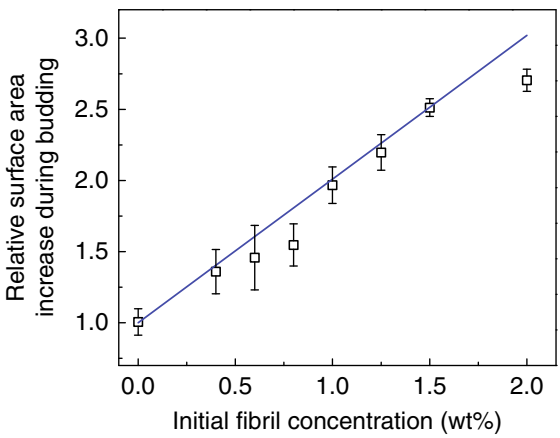

e

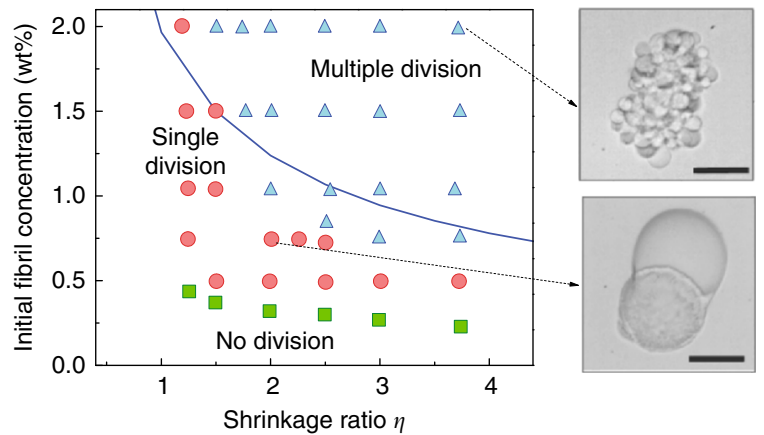

Fig. 3 Fibril networks control the number and size of daughter droplets. a The average diameter of daughter droplets is independent of the size of the mother droplet but increases linearly with increasing w/w interfacial tension. b After decomposition of fibril networks, the total surface area of the daughter droplets increases linearly with initial fibril concentration. c Radius and number of stabilized protrusions as a function of fibril concentration. Scale bars, $100 \mu \mathrm{m}$. $\mathbf{d}$ Diameter of stabilized daughter droplets as a function of the product of shrinkage ratio $(\eta)$ and initial fibril concentration $\left(C_{\text {fibril }}\right)$. e Phase diagram summarizing the effects of shrinkage ratio and initial fibril concentration on the division regimes of $\mathrm{w} / \mathrm{w}$ droplets loaded with protein nanofibrils. The initial diameter of the mother droplet was $250 \pm 20 \mu \mathrm{m}$. Inset: optical microscope images showing representative $\mathrm{w} / \mathrm{w}$ droplets in the multiple (top) and single (bottom) division regimes. Scale bars are $200 \mu \mathrm{m}$. The solid blue lines in a-e are predictions from Eq. (2). The size of daughter droplets, measured from 50 replicates, are expressed as (mean \pm s.d.)
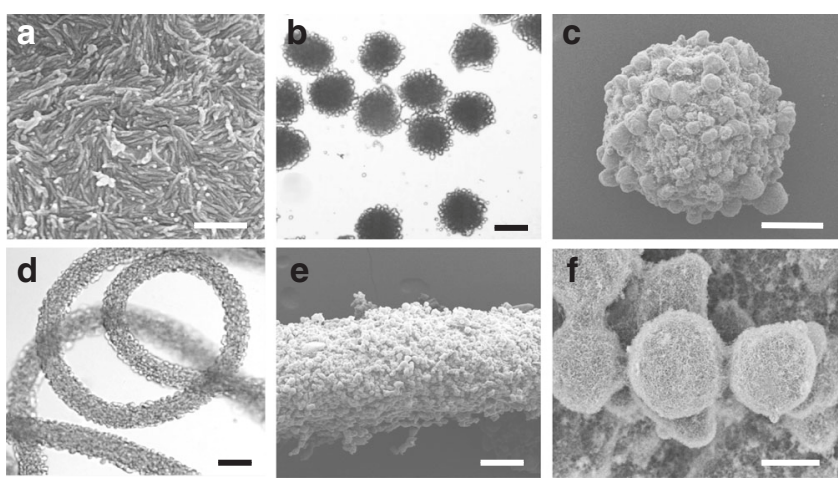

Fig. 4 Microfluidic fabrication of protein microparticles and microfibers with surface protrusions. a Scanning electron microscope (SEM) image of fibril networks. Scale bar, $500 \mathrm{~nm}$. b Optical microscope and c SEM images of microbeads with surface protrusions. d Optical microscope and e SEM images of a microfiber with $\mathbf{f}$ surface protrusions. Scale bars: $200 \mu \mathrm{m}(\mathbf{b}, \mathbf{d})$, $100 \mu \mathrm{m}(\mathbf{c}, \mathbf{e})$, and $10 \mu \mathrm{m}(\mathbf{f})$

of dehydration and the budding kinetics, the dextran-rich protrusions could be immobilized on the surface of the microparticles and microfibers, as shown by the optical and scanning electron microscope (SEM) images in Fig. 4. The physical dimensions of the microparticles and fibers as well as the sizes of the formed surface protrusions can be tuned by adjusting the osmotic pressure or the fibril concentration in the dispersed phase. This approach allows control of surface roughness 34,35 and design of surface patches ${ }^{36}$ of protein-based hydrogels in the all-aqueous environment, which will be of interest for the micropatterning of biomolecules and cells ${ }^{37}$ on the surface of biomaterials, such as cell-laden matrices, protein-delivery vehicles, and tissue engineering scaffolds ${ }^{38}$.

In this study we have suggested a possible route for achieving division of liquid droplets by exploiting synthetic protein fibrillization. Based on these results, we have further developed an all-aqueous platform for controlling the morphology of protein-rich gels, which could have important implications in tissue engineering and cell-mimicking studies.

\section{Methods}

Fabrication of fibril networks. An aqueous solution of chicken egg white was prepared by dissolving $2 \mathrm{wt} \%$ of the protein into a solution of $206 \mathrm{mM}$ hydrochloric acid ( $\mathrm{HCl}$, Aladdin Trade Co. Ltd) containing $2 \mathrm{mM}$ sodium chloride $\left(\mathrm{NaCl}\right.$, Sigma-Aldrich). The solution was incubated at $65^{\circ} \mathrm{C}$ for $70 \mathrm{~h}$ to induce polymerization of protein monomers into long fibrils. To achieve complete conversion from monomers to mature fibrils, the colloidal suspension was stirred at a centrifugal speed of $7 \times g$. The fibril suspension was diluted to $0.25-2 \mathrm{wt} \%$ by adding water. The dynamic viscosity of the suspension was measured using a microfluidic viscometer ( $\mu$ VISC, Rheosense, Inc.).

Fabrication of $\mathbf{w} / \mathbf{w}$ droplets. The emulsion phase was prepared by vortex mixing dextran T500 (Shanghai Ryon Biological Co., Ltd.) with the fibril suspension (see fabrication of fibril networks). The final concentration of dextran in the emulsion phase was 5-12 wt\%. The continuous phase was prepared by dissolving $8 \mathrm{wt} \%$ PEG $(\mathrm{Mw}=20,000$, Aladdin Trade Co., Ltd. $)$ in an aqueous solution of $10 \mathrm{mM} \mathrm{HCl}$.

$\mathrm{W} / \mathrm{w}$ emulsion droplets were generated by using an all-aqueous electrospray setup $^{27}$. The emulsion phase was charged positively $(\sim 2.5 \mathrm{kV})$, and subsequently sprayed into the continuous phase through a glass capillary with a nozzle diameter of $80 \mu \mathrm{m}$. A negatively charged metallic ring, with an enclosed circle of $4 \mathrm{~cm}$ in 
diameter, was positioned $1 \mathrm{~mm}$ beneath the capillary nozzle. The flow rate of the dispersed phase was maintained at $0.5 \mathrm{~mL} \mathrm{~h}^{-1}$.

Microscopy observation. The process of budding was monitored by using fluorescence microscopy (DMIL, LED, Fluo Leica): the dextran-rich phase was tagged by dissolving 0.3 wt $\%$ FITC-DEAE-Dextran $(\mathrm{Mw}=70,000$, Life Technology Co., Ltd.) into the droplet phase. In separate trials, the networks of lysozyme fibrils were stained by $15 \mu \mathrm{M} \mathrm{L}^{-1}$ ThT (Shanghai Ryon Biological Co., Ltd.), followed by incubation of the resultant mixture at $60^{\circ} \mathrm{C}$ for $15 \mathrm{~min}$. The fluorescence staining was excited by using a blue laser source and the images were captured in the green channels. We also obtained corresponding bright-field (or phase-contrast) images of the budding droplets for measuring the volume of the w/w droplets.

Osmolarity and interfacial tension measurements. Osmolarity of the emulsion phases before and after budding were measured using an osmometer (Model 3320, Advanced Instrument, Inc., USA). To determine the osmotic pressure between the droplet and continuous phases, we measured the osmolarity of dextran solutions before and after dehydration in the PEG-rich phase without addition of lysozyme fibrils, because the contribution of fibril networks to the osmolarity change of the emulsion phase can be ignored. After the splitting of droplets, the equilibrium concentration of dextran was determined from the phase diagram of dextran-PEG$\mathrm{H}_{2} \mathrm{O}$ reported in ref. ${ }^{39}$. The interfacial tension between different aqueous phases was measured using a spinning drop tensiometer (Krüss, Site 100). The protocol of measurement is illustrated in ref. 25

Fabrication of microparticles. An aqueous phase containing $2 \mathrm{wt} \%$ fibrils and $10 \%$ dextran T500 (droplet phase) was electrosprayed into the continuous phase consisting of an aqueous mixture of $25 \mathrm{wt} \%$ PEG $(\mathrm{Mw}=2000$, Aladdin Trade Co., Ltd.) and $0.1 \mathrm{wt} \%$ fibrils. At such a high concentration of PEG in the continuous phase, water was gradually extracted out of the droplet and the dextran-rich phase was solidified into solid particles. Glutaraldehyde ( $2.5 \mathrm{wt} \%$, Sigma) was added into the continuous phase to cross-link the protein nanofibrils. After reacting for $12 \mathrm{~h}$ at $37^{\circ} \mathrm{C}$, the microparticles with budding surfaces were formed.

Fabrication of microfibers. The above emulsion and continuous phases were separately injected through a co-flowing capillary microfluidic device, forming a stable dextran-in-PEG w/w jet. The flow rates of the dispersed and the outer continuous phases were maintained at $0.5 \mathrm{~mL} \mathrm{~h}^{-1}$ and $2 \mathrm{~mL} \mathrm{~h}^{-1}$, respectively. Subsequently, the $\mathrm{w} / \mathrm{w}$ jet was collected in a rotating culture dish $(\Phi=8 \mathrm{~cm}, 10$ $\mathrm{rpm}, \sim 0.004 \times g$ ). The culture dish contained a mixture solution of $30 \mathrm{wt} \%$ PEG $(\mathrm{Mw}=2000)$ and $2.5 \mathrm{wt} \%$ glutaraldehyde (Sigma). The w/w jet solidified into microfibers after incubation at $37^{\circ} \mathrm{C}$ for $4 \mathrm{~h}$.

Scanning electron microscopy. Microparticles and fibers were transferred into absolute ethanol to remove the remaining water. The particles and fibers were then immersed into liquid carbon dioxide and dried following the protocols of critical point drying ${ }^{40}$. The dried samples were sputtered with gold before imaging under scanning electron microscopy (Hitachi S4800 FEG, $5 \mathrm{kV}$ ).

Data availability. The authors declare that the data supporting the findings of this study are available within the paper and its Supplementary Information files and from the authors upon reasonable request.

Received: 13 December 2017 Accepted: 30 April 2018

Published online: 29 May 2018

\section{References}

1. Li, R. \& Murray, A. W. Feedback control of mitosis in budding yeast. Cell 66, 519-531 (1991).

2. Trajkovic, K. et al. Ceramide triggers budding of exosome vesicles into multivesicular endosomes. Science 319, 1244-1247 (2008).

3. Klopper, A. Cell swell. Nat. Phys. 11, 443-443 (2015).

4. Link, D. R. et al. Electric control of droplets in microfluidic devices. Angew. Chem. Int. Ed. 45, 2556-2560 (2006).

5. Baroud, C. N., De Saint Vincent, M. R. \& Delville, J. P. An optical toolbox for total control of droplet microfluidics. Lab. Chip 7, 1029-1033 (2007).

6. Yu, Y., Vroman, J. A., Bae, S. C. \& Granick, S. Vesicle budding induced by a pore-forming peptide. J. Am. Chem. Soc. 132, 195-201 (2009).

7. Baumgart, T., Hess, S. T. \& Webb, W. W. Imaging coexisting fluid domains in biomembrane models coupling curvature and line tension. Nature $\mathbf{4 2 5}$, 821-824 (2003).

8. Long, M. S., Cans, A. S. \& Keating, C. D. Budding and asymmetric protein microcompartmentation in giant vesicles containing two aqueous phases. $J$ Am. Chem. Soc. 130, 756-762 (2008).
9. Li, Y., Kusumaatmaja, H., Lipowsky, R. \& Dimova, R. Wetting-induced budding of vesicles in contact with several aqueous phases. J. Phys. Chem. B 116, 1819-1823 (2012).

10. Li, M., Huang, X. \& Mann, S. Spontaneous growth and division in selfreproducing inorganic colloidosomes. Small 10, 3291-3298 (2014).

11. Zwicker, D., Seyboldt, R., Weber, C. A., Hyman, A. A. \& Jülicher, F. Growth and division of active droplets provides a model for protocells. Nat. Phys. 13, 408-413 (2017).

12. Andes-Koback, M. \& Keating, C. D. Complete budding and asymmetric division of primitive model cells to produce daughter vesicles with different interior and membrane compositions. J. Am. Chem. Soc. 133, 9545-9555 (2011).

13. Fletcher, D. A. \& Mullins, R. D. Cell mechanics and the cytoskeleton. Nature 463, 485-492 (2010).

14. Mammoto, A. \& Ingber, D. E. Cytoskeletal control of growth and cell fate switching. Curr. Opin. Cell Biol. 21, 864-870 (2009).

15. Fogel, M. A. \& Waldor, M. K. A dynamic, mitotic-like mechanism for bacterial chromosome segregation. Genes Dev. 20, 3269-3282 (2006).

16. Wickstead, B. \& Gull, K. The evolution of the cytoskeleton. J. Cell Biol. 194, 513-525 (2011).

17. Lan, G., Daniels, B. R., Dobrowsky, T. M., Wirtz, D. \& Sun, S. X. Condensation of FtsZ filaments can drive bacterial cell division. Proc. Nat Acad. Sci. USA 106, 121-126 (2009).

18. Weiss, D. S. Bacterial cell division and the septal ring. Mol. Microbiol. 54, 588-597 (2004).

19. Strömqvist, J., Skoog, K., Daley, D. O., Widengren, J. \& Von Heijne, G. Estimating Z-ring radius and contraction in dividing Escherichia coli. Mol. Microbiol. 76, 151-158 (2010).

20. Kueh, H. Y. \& Mitchison, T. J. Structural plasticity in actin and tubulin polymer dynamics. Science 325, 960-963 (2009).

21. Slaughter, B. D., Smith, S. E. \& Li, R. Symmetry breaking in the life cycle of the budding yeast. Cold Spring Harb. Perspect. Boil. 1, a003384 (2009).

22. Sanchez, T., Chen, D. T., DeCamp, S. J., Heymann, M. \& Dogic, Z. Spontaneous motion in hierarchically assembled active matter. Nature 491, 431-434 (2012)

23. Long, M. S., Cans, A. S. \& Keating, C. D. Budding and asymmetric protein microcompartmentation in giant vesicles containing two aqueous phases. $J$. Am. Chem. Soc. 130, 756-762 (2008).

24. Walter, H. \& Brooks, D. E. Phase separation in cytoplasm, due to macromolecular crowding, is the basis for microcompartmentation. FEBS Lett. 361, 135-139 (1995).

25. Atefi, E., Mann, J. A. Jr \& Tavana, H. Ultralow interfacial tensions of aqueous two-phase systems measured using drop shape. Langmuir 30, 9691-9699 (2014).

26. Shimanovich, U. et al. Protein microgels from amyloid fibril networks. ACS Nano 9, 43-51 (2015).

27. Song, Y., Chan, Y. K., Ma, Q., Liu, Z. \& Shum, H. C. All-aqueous electrosprayed emulsion for templated fabrication of cytocompatible microcapsules. ACS Appl. Mater. Interfaces 7, 13925-13933 (2015).

28. Petkova, A. T. et al. A structural model for Alzheimer's $\beta$-amyloid fibrils based on experimental constraints from solid state NMR. Proc. Natl Acad. Sci. USA 99, 16742-16747 (2002).

29. Song, Y. et al. Fabrication of fibrillosomes from droplets stabilized by protein nanofibrils at all-aqueous interfaces. Nat. Commun. 7, 12934 (2016).

30. Arditty, S., Whitby, C. P., Binks, B. P., Schmitt, V. \& Leal-Calderon, F. Some general features of limited coalescence in solid-stabilized emulsions. Eur. Phys. J. E. 11, 273-281 (2003).

31. Zhu, J. \& Hayward, R. C. Hierarchically structured microparticles formed by interfacial instabilities of emulsion droplets containing amphiphilic block copolymers. Angew. Chem. 120, 2143-2146 (2008).

32. Shum, H. C., Varnell, J. \& Weitz, D. A. Microfluidic fabrication of water-inwater (w/w) jets and emulsions. Biomicrofluidics 6, 012808 (2012).

33. Ma, Q., Song, Y., Baier, G., Holtze, C. \& Shum, H. C. Osmo-solidification of all-aqueous emulsion with enhanced preservation of protein activity. J. Mater. Chem. B 4, 1213-1218 (2016).

34. Gittens, R. A. et al. The effects of combined micron-/submicron-scale surface roughness and nanoscale features on cell proliferation and differentiation. Biomaterials 32, 3395-3403 (2011).

35. Faia-Torres, A. B. et al. Differential regulation of osteogenic differentiation of stem cells on surface roughness gradients. Biomaterials 35, 9023-9032 (2014).

36. Meng, F. \& Zhong, Z. Polymersomes spanning from nano-to microscales: advanced vehicles for controlled drug delivery and robust vesicles for virus and cell mimicking. J. Phys. Chem. Lett. 2, 1533-1539 (2011).

37. Frampton, J. P., Tsuei, M., White, J. B., Abraham, A. T. \& Takayama, S Aqueous two-phase system-mediated antibody micropatterning enables multiplexed immunostaining of cell monolayers and tissues. Biotechnol. J. 10, 121-125 (2015) 
38. Hasirci, V. \& Kenar, H. Novel surface patterning approaches for tissue engineering and their effect on cell behavior. Nanomedicine 1, 73-90 (2006).

39. Nguyen, B. T., Nicolai, T. \& Benyahia, L. Stabilization of water-in-water emulsions by addition of protein particles. Langmuir 29, 10658-10664 (2013).

40. Steinbrecht, R. A. \& Zierold, K. (eds) Cryotechniques in Biological Electron Microscopy (Springer-Verlag, Berlin, 1987).

\section{Acknowledgements}

We are very grateful to Professor Michael Cates (Cambridge) and Professor L. Mahadevan (Harvard) for helpful discussions and for input into the theoretical analysis. This research was supported by the Early Career Scheme (HKU 707712P), the General Research Fund (HKU 719813E, 17304514, and 17306315) and the Collaborative Research Fund (C6004-14G) from the Research Grants Council of Hong Kong, the Major Research Plan (91434202) and the General Program (21476189/B060201) from the National Natural Science Foundation of China, the National Science Foundation of USA (IIP1456281), the Swiss National Science Foundation (T.C.T.M.), Peterhouse Cambridge (T.C.T.M.), the European Research Council under the European Union's Seventh Framework Program (FP7/2007-2013) through the ERC grant PhysProt (agreement no 337969), the Wellcome Trust (T.P.J.K.) and the Frances and Augustus Newman Foundation (T.P.J.K.).

\section{Author contributions}

Y.S. and T.C.T.M. analyzed the data, built the model, and wrote the paper. Y.S., Q.M., Z. L., and H.Y. designed and conducted the experiments. H.C.S., T.P.J.K., and S.T. supervised the project and wrote the paper.

\section{Additional information}

Supplementary Information accompanies this paper at https://doi.org/10.1038/s41467018-04510-3.

Competing interests: The authors declare no competing interests.

Reprints and permission information is available online at http://npg.nature.com/ reprintsandpermissions/

Publisher's note: Springer Nature remains neutral with regard to jurisdictional claims in published maps and institutional affiliations.

(c) Open Access This article is licensed under a Creative Commons Attribution 4.0 International License, which permits use, sharing, adaptation, distribution and reproduction in any medium or format, as long as you give appropriate credit to the original author(s) and the source, provide a link to the Creative Commons license, and indicate if changes were made. The images or other third party material in this article are included in the article's Creative Commons license, unless indicated otherwise in a credit line to the material. If material is not included in the article's Creative Commons license and your intended use is not permitted by statutory regulation or exceeds the permitted use, you will need to obtain permission directly from the copyright holder. To view a copy of this license, visit http://creativecommons.org/ licenses/by/4.0/.

(C) The Author(s) 2018 